\title{
Congenital Toxoplasmosis: Public Health Policy Concerns
}

Tazio Vanni, Rodrigo A. Ribeiro, Ivana S. Varella, Carisi A. Polanczyk, Benedito A. L. Fonseca and Ricardo Kuchenbecker Department of Social Medicine, Faculty of Medicine, Federal University of Rio Grande do Sul; Porto Alegre, RS, Brazil

Toxoplasmosis is caused by the parasite Toxoplasma gondii and is most often a benign disease. Two populations are at risk of severe disease, immunocompromised such as HIV-infected patients and fetuses or children with toxoplasmosis transmitted from their mothers via placenta. Congenital toxoplasmosis is rare on average, less than one case per 1,000 pregnancies, since mother-to-child transmission occurs only when infection is acquired for the first time during pregnancy [1]. Overall, about a third of infected mothers give birth to an infant with toxoplasmosis. Most children with congenital toxoplasmosis are developmentally normal but up to $4 \%$ die or have evidence of permanent neurological damage or bilateral visual impairment during the first years of life [2].

The birth prevalence of congenital toxoplasmosis ranges from 1/10,000 live births in Sweden and Massachusetts, USA, $3 / 10,000$ in Brazil to an estimate of 10/10,000 in France [3]. As well as its prevalence, the disease burden of congenital toxoplasmosis is important information for the development of evidence-based public health policies. Recently and for the first time, the burden of this disease was estimated by Havelaar et al.. They found that the disease burden of congenital toxoplasmosis in the Netherlands is about 620 (range 220-1900) disabilityadjusted life years (DALYs) per year, similar to that for salmonellosis (670 DALYs per year) [4].

Toxoplasma infection in pregnancy is usually asymptomatic and can only be detected by serological testing. The aim of prenatal serological screening for toxoplasmosis is to identify and treat maternal infection as soon as possible in order to prevent congenital toxoplasmosis. However, many aspects of surveillance and treatment of toxoplasmosis during pregnancy have remained controversial, since only observational studies with limited number of patients were available [2]. Uncertainty about the benefits of prenatal treatment as well as concerns about its adverse effects and the costs involved in a prenatal screening program have led to diverse public health policies including no screening (e.g. United Kingdom), neonatal screening (e.g. Massachusetts), and prenatal screening with monthly (e.g. France) or 3-monthly re-testing schedules (e.g. Brazil) [3].

Received on 5 December 2007; revised 4 April 2008.

Address for correspondence: Dr. Tazio Vanni. Departamento de Medicina Social, Faculdade de Medicina, Universidade Federal do Rio Grande do Sul. Rua Ramiro Barcelos, 2400, 2 andar. Zip code: 90035-003 - Porto Alegre, RS, Brazil. E-mail: tazio_vanni@yahoo.com.br. Fax: (55- 51) 3316-5620, Phone: (55-16) 3316-5621.

The Brazilian Journal of Infectious Diseases 2008;12(2):107. (C) 2008 by The Brazilian Journal of Infectious Diseases and Contexto Publishing. All rights reserved.
It is important to emphasize the results of the first metaanalysis assessing the effect of timing and type of prenatal treatment on mother-to-child transmission of infection and clinical manifestation before the age of one year, recently published by the SYROCOT study group. The authors found no evidence that prenatal treatment significantly reduces the risk of clinical manifestation of congenital toxoplasmosis (adjusted OR for treated vs. not treated 1.11, 95\% CI 0.61-2.02). Most of the limitations of this study were due to biases in the way the cohort studies were designed and undertaken, such as selection bias, and the absence of information on the consequences of intracranial lesions for subsequent development $[1,2,5]$. The fact that cohorts from America were excluded from this study also limits the extrapolation of its results to this continent. Despite these limitations, this study is the strongest evidence yet regarding prenatal treatment of toxoplasmosis. Only a large randomized controlled clinical trial would provide stronger evidence concerning this issue [1]. Nonetheless, taking into account the great number of patients and the high costs involved in a randomized clinical trial of this size, it is improbable that its results would be made available in a short term.

In concusion we firmly believe that the latest studies have raised the urgent need for us to discuss the following questions:

1. Do we have local data to estimate the burden of the disease and the economic impact of congenital toxoplasmosis to guide the decisions concerning public health policy?

2. Should we support a public health policy to prevent congenital toxoplasmosis by screening and treating pregnant women that has uncertain benefits, but related risks (e.g. adverse effects of the treatment, fetal loss due to amniocentesis) and costs [2]?

\section{References}

1. Thiétbaut R., Leroy V., Alioum A., et al. Biases in observational studies of the effect of prenatal treatment for congenital toxoplasmosis. Eur J Obstet Gynecol Reprod Biol $2006 ; 124: 3-9$.

2. The SYROCOT (Systematic Review on Congenital Toxoplasmosis) study group. Effectiveness of prenatal treatment for congenital toxoplasmosis: a meta-analysis of individual patients' data. Lancet 2007;369:115-22.

3. Gilbert R.E., Peckham C.S. Congenital Toxoplasmosis in the United Kingdom: to screen or not to screen? J Med Screen 2002;9(3):135-41.

4. Havelaar A.H., Kemmeren J.M., Kortbeek L.M. Disease burden of congenital toxoplasmosis. Clin Infect Dis 2007;44:1467-74.

5. Gilbert R.E., Gras L., Wallon M., et al. Effect of prenatal treatment on mother to child transmission of Toxoplasma gondii: retrospective cohort study of 554 mother-child pairs in Lyon, France. Int J Epidemiol 2001;30:1303-8. 